\title{
Adaptación peruana de la Lista de Verificación de Síntomas Pediátricos como instrumento de tamizaje de problemas conductuales y emocionales en niños, niñas y adolescentes.
}

Peruvian adaptation of the Pediatric Symptoms Checklist as a screening tool for behavioral and emotional problems in children and adolescents.

\author{
Carla P. Cortez-Vergara ${ }^{1,2}$, July Caballero-Peralta ${ }^{1}$, Johann M. Vega-Dienstmaier ${ }^{1}$
}

Identificación ORCID:

Carla P. Cortez-Vergara: https://orcid.org/0000-0002-7068-1927

July Caballero-Peralta: https://orcid.org/0000-0001-5530-1220

Johann M. Vega-Dienstmaier: https://orcid.org/0000-0002-5686-4014

\section{RESUMEN}

Objetivo: Adaptación cultural para uso en la población peruana de la Lista de Verificación de Síntomas Pediátricos (Pediatric Symptoms Checklist, PSC-17), un cuestionario que asiste en la identificación de problemas emocionales y conductuales en individuos de 6 a 17 años. Método: Sobre la base de los textos originales del PSC-17 en inglés y español y de la versión chilena, un comité de cinco expertos llevó a cabo la adaptación de los 17 ítems para la versión de padres y cuidadores y para la de adolescentes. Resultados: Algunos ítems se conservaron idénticos a una de las dos versiones en español; otros se mantuvieron similares a la versión chilena y algunos tuvieron que modificarse ligeramente para ajustarse al idioma español de uso en Perú. Conclusiones: La versión peruana final del PSC-17 facilitaría la detección de problemas de salud mental en niños y adolescentes en nuestro país, aun cuando es necesario llevar a cabo posteriormente estudios sobre sus propiedades psicométricas.

PALABRAS CLAVE: Trastornos de la conducta infantil, salud mental, tamizaje masivo, niños, conducta del adolescente.

\section{SUMMARY}

Objective: Adaptation to Peruvian population of the Pediatric Symptoms Checklist (PSC-17), a questionnaire that assists in the identification of emotional and behavioral problems in children and adolescents between 6 to 17 years. Method: On the basis of the original texts of PSC-17 in English and Spanish and the Chilean version, a committee of five experts proceeded to the adaptation of the 17 items for parents and caregivers and for adolescents. Results: Some items were kept identical to one of the two revised Spanish versions; others remained similar to the Chilean version, and some had to be slightly modified to match Peruvian Spanish. Conclusions: The final Peruvian version of the PSC-17 would facilitate the detection of mental health problems in children and adolescents in our country, even though it is still necessary to subsequently carry out pertinent studies of its psychometric properties.

KEYWORDS: Child behavior disorders, mental health, mass screening, child, adolescent behavior.

\footnotetext{
Universidad Peruana Cayetano Heredia, Facultad de Medicina Alberto Hurtado. Lima, Perú.

2 Clínica Anglo Americana. Lima, Perú.
} 


\section{INTRODUCCIÓN}

La afectación de la salud mental de niños, niñas y adolescentes en el contexto de la pandemia de COVID-19 ha sido ampliamente descrita $(1,2)$. Las distintas restricciones, el confinamiento prolongado, el impacto socioeconómico y los cambios en la rutina asociados a las medidas para evitar la propagación del virus han generado mayor vulnerabilidad para el desarrollo de problemas de salud mental en esta población. Estas circunstancias, aunadas a otras adversidades y en ausencia de apoyo adecuado por parte de los adultos, puede devenir en respuestas al estrés tóxico que afectan acumulativamente la salud física y mental del niño (3). En nuestro país, los niños, niñas y adolescentes tuvieron que sobrellevar un confinamiento mayor a 6 meses en el cual se limitaron sus posibilidades de realizar actividades recreativas, deportivas, sociales, culturales, educativas y familiares. Por este motivo, la Dirección de Salud Mental propuso la tarea de evaluar de manera objetiva este impacto mediante una encuesta en línea a escala nacional. En el marco de dicha encuesta ("La salud mental de niñas, niños y adolescentes en el contexto de la Covid-19. Estudio en línea - Perú 2020"), se requería contar con un instrumento de tamizaje de problemas de salud mental en la población infantojuvenil.

El uso de instrumentos de tamizaje puede ayudar a los profesionales de salud a reducir muchas de las barreras para la detección e incrementar la comunicación, identificación e intervención de problemas de salud mental en niños(as) (2). Sin embargo, no se cuenta con instrumento validado en nuestro país, con excepción del Child Behaviour Checklist (CBCL), utilizado previamente por el Instituto Nacional de Salud Mental (4). Si bien este instrumento cuenta con amplio respaldo en la comunidad científica y ha sido investigado en numerosos estudios dando cuenta de su validez y confiabilidad $(5,6)$, el gran número de preguntas supone una dificultad para su contestación mediante una encuesta virtual.

Luego de una revisión de la literatura, se seleccionó el instrumento "Lista de Verificación de Síntomas Pediátricos" (PSC, por sus siglas en inglés) que es un cuestionario breve que apoya la identificación de problemas emocionales y conductuales en niños, niñas y adolescentes de 6 a 17 años. El PSC cubre una amplia gama de problemas emocionales y conductuales y puede ser usado con facilidad por personal de salud del primer nivel de atención (7) e incluso en entornos escolares (8). El cuestionario original consta de 35 ítems, pero se cuenta con la versión corta, de 17 ítems (PSC-17) que aborda tres dimensiones o factores: problemas internalizantes, problemas externalizantes y problemas atencionales. Ambos cuestionarios cuentan con versiones para adolescentes y para padres y cuidadores. El PSC ha sido traducido a múltiples idiomas y utilizado hace más de una década en numerosos países en el contexto clínico y de investigación $(9,10)$. En el idioma español se cuenta con versiones española y chilena (7). Se ha demostrado una elevada confiabilidad, con una consistencia interna de 0,89 y de test-retest de 0,85 ; igualmente, el análisis factorial confirmatorio respalda el modelo original de tres factores $(8,11)$. El PSC-17 ha sido utilizado y validado en una muestra chilena de 5177 estudiantes de primer grado, encontrándose también el modelo de tres factores mediante análisis factorial (12). Las principales ventajas del PSC-17 radican en la brevedad de su aplicación, su eficiencia para el tamizaje universal y la gratuidad de su uso (7).

El presente trabajo tuvo como objetivo realizar la adaptación al contexto peruano del instrumento de tamizaje de problemas conductuales en niños y adolescentes "Lista de Verificación de Síntomas Pediátricos" (PSC) de manera que la versión peruana sea culturalmente equivalente, resulte aplicable en nuestro país y aceptable para la población objetivo (13).

\section{MATERIAL Y MÉTODOS}

Es un estudio de adaptación cultural de un instrumento para identificación de manifestaciones emocionales y conductuales en niños y adolescentes.

Antes de iniciar la adaptación, se solicitó la autorización al desarrollador del instrumento PSC mediante el correo provisto en la página web oficial (7).

Con base en los cuestionarios existentes en español: Cuestionario en español disponible en la página web del desarrollador y cuestionario chileno (versión correspondiente a la utilizada por la Junta Nacional de Auxilio Escolar y Becas del Ministerio de Educación de Chile en el contexto del Programa Habilidades para la Vida), los investigadores, elaboraron una versión peruana preliminar, tanto para la versión de padres y cuidadores como la de adolescentes. Ambas fueron evaluadas, discutidas y mejoradas por un comité evaluador.

El cuestionario original en inglés, el cuestionario original en español (7), el cuestionario chileno (14) y la versión peruana preliminar fueron remitidas a un comité evaluador para su revisión mediante juicio de expertos. 
Dicho comité estuvo conformado por 4 psiquiatras con más de 5 años de experiencia que incluyo a 3 psiquiatras de niños y adolescentes y un psiquiatra de adultos con amplia experiencia en investigación y una enfermera especializada en salud mental. Todos los profesionales tuvieron experiencia clínica basada en su labor en establecimientos de referencia a nivel nacional y también en investigación y tres de ellos tuvieron nivel de competencia avanzada en el idioma inglés. Los jueces realizaron la lectura y análisis de los cuatro cuestionarios en las versiones de padres y cuidadores y de adolescentes y discutieron las diferencias entre ellos y la connotación de cada uno de los ítems en el contexto peruano. Además, mediante discusión y consenso, elaboraron una propuesta para cada uno de los ítems de manera que se mantuviera la equivalencia conceptual y técnica con la versión original y teniendo en cuenta su aplicabilidad en las diferentes regiones del país. El mismo procedimiento fue realizado para las instrucciones y para las opciones de respuesta.

\section{RESULTADOS}

Las principales observaciones y aportes emitidos por el comité evaluador para las instrucciones, ítems y opciones de respuesta se describen con detalle en las tablas 1, 2, 3, 4 y 5.

\section{Versión para padres y cuidadores}

En relación con las instrucciones se optó por que sean breves y directas tal como la versión en inglés. Los ítems 4 (preocupación) y 8 (distraibilidad) se han mantenido igual en la versión peruana que en las otras versiones en español por considerarlas claras y comprensibles. Los ítems 1 (tristeza), 2 (desesperanza), 6 (intranquilidad), 7 (soñar despierto) y 9 (concentración) se mantuvieron iguales a la versión chilena ya que esta se consideró más clara y comprensible para el contexto peruano.

Los ítems 11 (peleas) y 16 (no compartir) son los mismos que la versión original en español al ser considerados más neutros para abordar esa característica. El resto de ítems presentan pequeñas modificaciones respecto a la versión original en español o la versión chilena para usar expresiones más comúnmente usadas en nuestro contexto. Los ítems 13 (empatía) y 15 (culpar a otros) presentan un ligero cambio respecto a las versiones original y chilena. Los ítems 3 (autoestima) y 14 (molestar a otros) se acercan más a la versión original en español; mientras que los ítems 5 (anhedonia) y 17 (coge cosas ajenas) son más parecidos a la versión chilena. El ítem 10 (hiperactividad) y 12 (desobediencia) combinan el contenido de la versión original y la chilena.

Tabla 1. Síntesis de la evaluación de la sección "Instrucciones" (versión para padres y cuidadores) por parte del comité evaluador.

\begin{tabular}{|c|c|c|c|c|c|}
\hline $\begin{array}{l}\text { Original en } \\
\text { inglés }^{\mathrm{a}}\end{array}$ & Original en español $^{\mathrm{b}}$ & $\begin{array}{c}\text { Versión } \\
\text { Chilena } \\
\text { JUNAEB }\end{array}$ & $\begin{array}{c}\text { Versión } \\
\text { Peruana } \\
\text { Preliminar }\end{array}$ & Observaciones & $\begin{array}{c}\text { Versión } \\
\text { peruana final }\end{array}$ \\
\hline $\begin{array}{l}\text { Please mark } \\
\text { under the } \\
\text { heading that } \\
\text { best describes } \\
\text { your child: }\end{array}$ & $\begin{array}{l}\text { La salud física y emocional son } \\
\text { importantes para cada niño. } \\
\text { Los padres son los primeros } \\
\text { que notan un problema de } \\
\text { la conducta emocional o del } \\
\text { aprendizaje de su hijo(a). Ud. } \\
\text { puede ayudar a su hijo(a) a } \\
\text { obtener el mejor cuidado de su } \\
\text { doctor por medio de contestar } \\
\text { estas preguntas. Favor de } \\
\text { indicar cuál frase describe a su } \\
\text { hijo(a). Indique cual síntoma } \\
\text { mejor describe a su hijo/a: }\end{array}$ & $\begin{array}{l}\text { No } \\
\text { especifica } \\
\text { indicaciones. }\end{array}$ & $\begin{array}{l}\text { Por favor, } \\
\text { indique lo que } \\
\text { mejor describa } \\
\text { a su hijo(a): }\end{array}$ & $\begin{array}{l}\text { El comité } \\
\text { consideró por } \\
\text { unanimidad que } \\
\text { las instrucciones } \\
\text { se mantengan lo } \\
\text { más similares } \\
\text { posible a la } \\
\text { versión original } \\
\text { en inglés por } \\
\text { ser más claras y } \\
\text { directas. }\end{array}$ & $\begin{array}{l}\text { Por favor, } \\
\text { indique lo que } \\
\text { mejor describa } \\
\text { a su hijo(a): }\end{array}$ \\
\hline
\end{tabular}

${ }^{a}$ Cuestionario tomado de la página web del desarrollador, disponible en: https://www.massgeneral.org/assets/MGH/pdf/psychiatry/psc/ psc-17-english.pdf

${ }^{\mathrm{b}}$ Cuestionario tomado de la página web del desarrollador, disponible en: https://www.massgeneral.org/assets/MGH/pdf/psychiatry/psc/ psc-17-spanish.pdf

${ }^{c}$ Versión del cuestionario correspondiente a la utilizada por la Junta Nacional de Auxilio Escolar y Becas del Ministerio de Educación de Chile en el contexto del Programa Habilidades para la Vida. 
Tabla 2. Síntesis de la evaluación de los ítems del instrumento PSC (versión para padres y cuidadores) por parte del comité evaluador.

\begin{tabular}{|c|c|c|c|c|c|c|}
\hline $\begin{array}{c}\text { Ítem } \\
N^{\circ}\end{array}$ & $\begin{array}{c}\text { Original en } \\
\text { inglés }^{\mathrm{a}}\end{array}$ & $\begin{array}{c}\text { Original en } \\
\text { español }^{\mathrm{b}}\end{array}$ & $\begin{array}{l}\text { Versión } \\
\text { Chilena } \\
\text { JUNAEB }^{\mathrm{c}}\end{array}$ & $\begin{array}{l}\text { Versión Peruana } \\
\text { Preliminar }\end{array}$ & Observaciones & $\begin{array}{c}\text { Versión peruana } \\
\text { final }\end{array}$ \\
\hline 1 & $\begin{array}{l}\text { Feels sad, } \\
\text { unhappy }\end{array}$ & $\begin{array}{l}\text { Se siente triste, } \\
\text { infeliz }\end{array}$ & Se siente triste & Se siente triste & $\begin{array}{l}\text { Se considera mantener } \\
\text { la versión chilena } \\
\text { y omitir la palabra } \\
\text { "infeliz" } \\
\text { ese término norque } \\
\text { comúnmente usado } \\
\text { para expresar un estado } \\
\text { de ánimo. }\end{array}$ & Se siente triste \\
\hline 2 & Feels hopeless & $\begin{array}{l}\text { Se siente sin } \\
\text { esperanzas }\end{array}$ & $\begin{array}{l}\text { Se siente } \\
\text { pesimista/piensa } \\
\text { que las cosas } \\
\text { son difíciles y le } \\
\text { van a salir mal }\end{array}$ & $\begin{array}{l}\text { Se siente pesimista/ } \\
\text { piensa que las cosas } \\
\text { son difíciles y le } \\
\text { van a salir mal }\end{array}$ & $\begin{array}{l}\text { Se considera mantener } \\
\text { la versión chilena por } \\
\text { considerarla clara y } \\
\text { comprensible. }\end{array}$ & $\begin{array}{l}\text { Se siente } \\
\text { pesimista/piensa } \\
\text { que las cosas son } \\
\text { difíciles y le van a } \\
\text { salir mal }\end{array}$ \\
\hline 3 & $\begin{array}{l}\text { Is down on } \\
\text { self }\end{array}$ & $\begin{array}{l}\text { Se siente mal de } \\
\text { sí mismo(a) }\end{array}$ & $\begin{array}{l}\text { Se siente poca } \\
\text { cosa }\end{array}$ & $\begin{array}{l}\text { No se siente bien } \\
\text { consigo mismo(a) }\end{array}$ & $\begin{array}{l}\text { El ítem original se } \\
\text { refiere a la evaluación } \\
\text { en relación con uno } \\
\text { mismo. El término } \\
\text { "poca cosa" puede } \\
\text { no ser muy utilizado, } \\
\text { además el comité } \\
\text { considera que tiene una } \\
\text { connotación negativa y } \\
\text { puede sonar muy fuerte } \\
\text { para su uso en niños. }\end{array}$ & $\begin{array}{l}\text { Se siente mal } \\
\text { consigo mismo }\end{array}$ \\
\hline 4 & Worries a lot & $\begin{array}{l}\text { Se preocupa } \\
\text { mucho }\end{array}$ & $\begin{array}{l}\text { Se preocupa } \\
\text { mucho }\end{array}$ & Se preocupa mucho & $\begin{array}{l}\text { Se considera mantener } \\
\text { la versión previa por } \\
\text { considerarla clara y } \\
\text { comprensible. }\end{array}$ & $\begin{array}{l}\text { Se preocupa } \\
\text { mucho }\end{array}$ \\
\hline 5 & $\begin{array}{l}\text { Seems to be } \\
\text { having less } \\
\text { fun }\end{array}$ & $\begin{array}{l}\text { Parece divertirse } \\
\text { menos }\end{array}$ & Se aburre & $\begin{array}{l}\text { Parece que no le } \\
\text { divierten las cosas }\end{array}$ & $\begin{array}{l}\text { Se considera añadir } \\
\text { "fácilmente" a la } \\
\text { versión chilena para } \\
\text { hacer hincapié en } \\
\text { que se trata de un } \\
\text { aburrimiento mayor a } \\
\text { lo normal o esperado } \\
\text { en un niño sin } \\
\text { patología. Además, } \\
\text { porque muchos niños } \\
\text { pueden expresar } \\
\text { aburrimiento sin que } \\
\text { ello sea indicador de } \\
\text { algún problema, más } \\
\text { aún en el contexto del } \\
\text { confinamiento. }\end{array}$ & $\begin{array}{l}\text { Se aburre } \\
\text { fácilmente }\end{array}$ \\
\hline 6 & $\begin{array}{l}\text { Fidgety, } \\
\text { unable to sit } \\
\text { still }\end{array}$ & $\begin{array}{l}\text { Es inquieto(a), } \\
\text { incapaz de } \\
\text { sentarse } \\
\text { tranquilo(a) }\end{array}$ & $\begin{array}{l}\text { Le cuesta estar } \\
\text { tranquilo }\end{array}$ & $\begin{array}{l}\text { Le cuesta estar } \\
\text { tranquilo(a) }\end{array}$ & $\begin{array}{l}\text { Se considera mantener } \\
\text { la versión chilena por } \\
\text { considerarla clara y } \\
\text { comprensible. }\end{array}$ & $\begin{array}{l}\text { Le cuesta estar } \\
\text { tranquilo(a) }\end{array}$ \\
\hline 7 & $\begin{array}{l}\text { Daydreams } \\
\text { too much }\end{array}$ & $\begin{array}{l}\text { Sueña despierto } \\
\text { demasiado }\end{array}$ & $\begin{array}{l}\text { Sueña despierto/ } \\
\text { está en su propio } \\
\text { mundo }\end{array}$ & $\begin{array}{l}\text { Sueña despierto(a)/ } \\
\text { está en su propio } \\
\text { mundo }\end{array}$ & $\begin{array}{l}\text { Se considera mantener } \\
\text { la versión chilena por } \\
\text { considerarla clara y } \\
\text { comprensible. }\end{array}$ & $\begin{array}{l}\text { Sueña } \\
\text { despierto(a)/ } \\
\text { está en su propio } \\
\text { mundo }\end{array}$ \\
\hline
\end{tabular}


Tabla 2 (cont). Síntesis de la evaluación de los ítems del instrumento PSC (versión para padres y cuidadores) por parte del comité evaluador.

\begin{tabular}{|c|c|c|c|c|c|c|}
\hline $\begin{array}{c}\text { Ítem } \\
\mathrm{N}^{\circ}\end{array}$ & $\begin{array}{c}\text { Original en } \\
\text { inglés }^{\mathrm{a}}\end{array}$ & $\begin{array}{c}\text { Original en } \\
\text { español }^{b}\end{array}$ & $\begin{array}{c}\text { Versión } \\
\text { Chilena } \\
\text { JUNAEB }^{c}\end{array}$ & $\begin{array}{c}\text { Versión Peruana } \\
\text { Preliminar }\end{array}$ & Observaciones & $\begin{array}{c}\text { Versión peruana } \\
\text { final }\end{array}$ \\
\hline 8 & $\begin{array}{l}\text { Distracted } \\
\text { easily }\end{array}$ & $\begin{array}{l}\text { Se distrae } \\
\text { fácilmente }\end{array}$ & $\begin{array}{l}\text { Se distrae } \\
\text { fácilmente }\end{array}$ & $\begin{array}{l}\text { Se distrae } \\
\text { fácilmente }\end{array}$ & $\begin{array}{l}\text { Se considera mantener } \\
\text { la versión previa por } \\
\text { considerarla clara y } \\
\text { comprensible. }\end{array}$ & $\begin{array}{l}\text { Se distrae } \\
\text { fácilmente }\end{array}$ \\
\hline 9 & $\begin{array}{l}\text { Has trouble } \\
\text { concentrating }\end{array}$ & $\begin{array}{l}\text { Tiene problemas } \\
\text { para concentrarse }\end{array}$ & $\begin{array}{l}\text { Le cuesta } \\
\text { concentrarse }\end{array}$ & $\begin{array}{l}\text { Le cuesta } \\
\text { concentrarse }\end{array}$ & $\begin{array}{l}\text { Se considera mantener } \\
\text { la versión chilena por } \\
\text { considerarla clara y } \\
\text { comprensible. }\end{array}$ & $\begin{array}{l}\text { Le cuesta } \\
\text { concentrarse }\end{array}$ \\
\hline 10 & $\begin{array}{l}\text { Acts as if } \\
\text { driven by a } \\
\text { motor }\end{array}$ & $\begin{array}{l}\text { Es muy activo(a), } \\
\text { tiene mucha } \\
\text { energía }\end{array}$ & Es inquieto(a) & Es muy inquieto(a) & $\begin{array}{l}\text { Se considera que, } \\
\text { de mantenerse } \\
\text { únicamente como } \\
\text { "es inquieto", sería } \\
\text { muy similar al ítem } 6 . \\
\text { Se mantiene el ítem } \\
\text { original en español, } \\
\text { pero se da connotación } \\
\text { de mayor intensidad } \\
\text { r e e m p l a z a n d o } \\
\text { "mucha energía" por } \\
\text { "demasiada energía" } \\
\text { porque "mucha } \\
\text { energía" puede ser } \\
\text { una cualidad esperada } \\
\text { en un niño o } 11 \\
\text { considerada como algo } \\
\text { "normal" o parte del } \\
\text { desarrollo infantil. }\end{array}$ & $\begin{array}{l}\text { Es muy } \\
\text { inquieto(a), tiene } \\
\text { demasiada energía }\end{array}$ \\
\hline 11 & $\begin{array}{l}\text { Fights with } \\
\text { other children }\end{array}$ & $\begin{array}{l}\text { Pelea con otros } \\
\text { niños }\end{array}$ & $\begin{array}{l}\text { Pelea con otros/ } \\
\text { as compañeros/ } \\
\text { as }\end{array}$ & $\begin{array}{l}\text { Pelea con otros } \\
\text { niños(as) }\end{array}$ & $\begin{array}{l}\text { Se considera mantener } \\
\text { la versión original } \\
\text { en español, pero } \\
\text { sustituyendo "otros } \\
\text { compañeros" por } \\
\text { "otros niños(as)" ya } \\
\text { que es más general } \\
\text { y no induce a pensar } \\
\text { únicamente en } \\
\text { entornos escolares en } \\
\text { un contexto en el cual } \\
\text { los niños han dejaron } \\
\text { de asistir a clases } \\
\text { presenciales. }\end{array}$ & $\begin{array}{l}\text { Pelea con otros } \\
\text { niños(as) }\end{array}$ \\
\hline 12 & $\begin{array}{l}\text { Does not } \\
\text { listen to rules }\end{array}$ & $\begin{array}{l}\text { No obedece las } \\
\text { reglas }\end{array}$ & $\begin{array}{l}\text { Ignora las } \\
\text { órdenes que le } \\
\text { dan }\end{array}$ & $\begin{array}{l}\text { No sigue las } \\
\text { normas/reglas }\end{array}$ & $\begin{array}{l}\text { Se reformula la versión } \\
\text { original en español para } \\
\text { expresar que el ítem } \\
\text { no trata únicamente } \\
\text { de obedecer reglas, } \\
\text { sino de no seguir } \\
\text { órdenes entendidas } \\
\text { como mandatos o } \\
\text { indicaciones además } \\
\text { de cumplir las reglas. }\end{array}$ & $\begin{array}{l}\text { No sigue las } \\
\text { órdenes/reglas }\end{array}$ \\
\hline
\end{tabular}


Adaptación peruana de la Lista de Verificación de Síntomas Pediátricos (PSC).

Tabla 2 (cont). Síntesis de la evaluación de los ítems del instrumento PSC (versión para padres y cuidadores) por parte del comité evaluador.

\begin{tabular}{|c|c|c|c|c|c|c|}
\hline $\begin{array}{c}\text { Ítem } \\
\mathbf{N}^{\circ}\end{array}$ & $\begin{array}{c}\text { Original en } \\
\text { inglés }^{\mathrm{a}}\end{array}$ & $\begin{array}{c}\text { Original en } \\
\text { español }^{\text {b }}\end{array}$ & $\begin{array}{c}\text { Versión } \\
\text { Chilena } \\
\text { JUNAEB }^{\mathbf{c}}\end{array}$ & $\begin{array}{l}\text { Versión Peruana } \\
\text { Preliminar }\end{array}$ & Observaciones & $\begin{array}{c}\text { Versión peruana } \\
\text { final }\end{array}$ \\
\hline 13 & $\begin{array}{l}\text { Does not } \\
\text { understand } \\
\text { other people's } \\
\text { feelings }\end{array}$ & $\begin{array}{l}\text { No comprende } \\
\text { los sentimientos } \\
\text { de otros }\end{array}$ & $\begin{array}{l}\text { No comprende } \\
\text { los sentimientos } \\
\text { de otros }\end{array}$ & $\begin{array}{l}\text { No comprende los } \\
\text { sentimientos de } \\
\text { otros }\end{array}$ & $\begin{array}{l}\text { Se mantiene la versión } \\
\text { original en español y la } \\
\text { chilena con un cambio } \\
\text { sutil en el fraseo que } \\
\text { es una expresión más } \\
\text { comúnmente usada. }\end{array}$ & $\begin{array}{l}\text { No comprende los } \\
\text { sentimientos de } \\
\text { los demás }\end{array}$ \\
\hline 14 & Teases others & $\begin{array}{l}\text { Molesta o se } \\
\text { burla de otros }\end{array}$ & $\begin{array}{l}\text { Molesta a los } \\
\text { demás }\end{array}$ & $\begin{array}{l}\text { Molesta o se burla } \\
\text { de otros }\end{array}$ & $\begin{array}{l}\text { Se mantiene la versión } \\
\text { original en español y la } \\
\text { chilena con un cambio } \\
\text { sutil en el fraseo que } \\
\text { es una expresión más } \\
\text { comúnmente usada } \\
\text { y para mantener } \\
\text { congruencia con el } \\
\text { ítem previo. }\end{array}$ & $\begin{array}{l}\text { Molesta o se burla } \\
\text { de los demás }\end{array}$ \\
\hline 15 & $\begin{array}{l}\text { Blames others } \\
\text { for his/her } \\
\text { troubles }\end{array}$ & $\begin{array}{l}\text { Culpa a otros por } \\
\text { sus problemas }\end{array}$ & $\begin{array}{l}\text { Culpa a } \\
\text { otros por sus } \\
\text { problemas }\end{array}$ & $\begin{array}{l}\text { Culpa a otros por } \\
\text { sus problemas }\end{array}$ & $\begin{array}{l}\text { Se mantiene la versión } \\
\text { original en español y la } \\
\text { chilena con un cambio } \\
\text { sutil en el fraseo que } \\
\text { es una expresión más } \\
\text { comúnmente usada } \\
\text { y para mantener } \\
\text { congruencia con el } \\
\text { ítem previo. }\end{array}$ & $\begin{array}{l}\text { Culpa a los demás } \\
\text { de sus problemas }\end{array}$ \\
\hline 16 & $\begin{array}{l}\text { Refuses to } \\
\text { share }\end{array}$ & $\begin{array}{l}\text { Se niega a } \\
\text { compartir }\end{array}$ & Es egoísta & $\begin{array}{l}\text { No comparte con } \\
\text { los demás }\end{array}$ & $\begin{array}{l}\text { Se prefirió no colocar } \\
\text { "compartir con los } \\
\text { demás" porque se } \\
\text { consideró que en } \\
\text { nuestro medio esa } \\
\text { forma de expresión } \\
\text { corresponde a } \\
\text { "compartir momentos" } \\
\text { generalmente con otras } \\
\text { personas (por ejemplo, } \\
\text { salir juntos, comer } \\
\text { juntos y actividades } \\
\text { similares). Se omitió } \\
\text { el término "egoísta" } \\
\text { porque implica } \\
\text { mucha subjetividad } \\
\text { e interpretación y } \\
\text { la redacción final, } \\
\text { coincidente con la } \\
\text { versión original en } \\
\text { español, es considerada } \\
\text { como más neutra o } \\
\text { imparcial. }\end{array}$ & $\begin{array}{l}\text { Se niega a } \\
\text { compartir }\end{array}$ \\
\hline
\end{tabular}


Tabla 2 (cont). Síntesis de la evaluación de los ítems del instrumento PSC (versión para padres y cuidadores) por parte del comité evaluador.

\begin{tabular}{|c|c|c|c|c|c|c|}
\hline $\begin{array}{c}\text { Ítem } \\
\mathrm{N}^{\circ}\end{array}$ & $\begin{array}{l}\text { Original en } \\
\text { inglés }^{\mathrm{a}}\end{array}$ & $\begin{array}{c}\text { Original en } \\
\text { español }^{\text {bs }}\end{array}$ & $\begin{array}{c}\text { Versión } \\
\text { Chilena } \\
\text { JUNAEB }^{\mathbf{c}}\end{array}$ & $\begin{array}{l}\text { Versión Peruana } \\
\text { Preliminar }\end{array}$ & Observaciones & $\begin{array}{c}\text { Versión peruana } \\
\text { final }\end{array}$ \\
\hline 17 & $\begin{array}{l}\text { Takes things } \\
\text { that do not } \\
\text { belong to him/ } \\
\text { her }\end{array}$ & $\begin{array}{l}\text { Toma cosas que } \\
\text { no le pertenecen }\end{array}$ & $\begin{array}{l}\text { Toma cosas } \\
\text { ajenas }\end{array}$ & Coge cosas ajenas & $\begin{array}{l}\text { Se propuso el término } \\
\text { "agarra" pero el } \\
\text { término "coge" tiene } \\
\text { una connotación más } \\
\text { definitiva o permanente } \\
\text { (por ejemplo, "coge } \\
\text { plata"), además de } \\
\text { que es una palabra } \\
\text { fre c u e n t e m e n t e } \\
\text { utilizada en el } \\
\text { contexto peruano en } \\
\text { comparación a "tomar" } \\
\text { (objetos). Se reformuló } \\
\text { el ítem. }\end{array}$ & Coge cosas ajenas \\
\hline & $\begin{array}{l}\text { Does your } \\
\text { child have any } \\
\text { emotional or } \\
\text { behavioral } \\
\text { problems for } \\
\text { which she/he } \\
\text { needs help? }\end{array}$ & $\begin{array}{l}\text { ¿Tiene su hijo(a) } \\
\text { algún problema } \\
\text { emocional o del } \\
\text { comportamiento } \\
\text { para el cual } \\
\text { necesita ayuda? }\end{array}$ & $\begin{array}{l}\text { Ausente en la } \\
\text { versión chilena }\end{array}$ & $\begin{array}{l}\text { ¿Tiene su hijo(a) } \\
\text { algún problema } \\
\text { emocional o del } \\
\text { comportamiento } \\
\text { para el cual } \\
\text { necesita ayuda? }\end{array}$ & $\begin{array}{l}\text { Todos los evaluadores } \\
\text { consideraron esta } \\
\text { pregunta como } \\
\text { importante } \\
\text { pertinente a pesar de } \\
\text { no estar incluida en } \\
\text { la versión chilena. } \\
\text { Por unanimidad se } \\
\text { consideró mantenerla } \\
\text { igual que en la versión } \\
\text { original en español. }\end{array}$ & $\begin{array}{l}\text { ¿Tiene su hijo(a) } \\
\text { algún problema } \\
\text { emocional o del } \\
\text { comportamiento } \\
\text { para el cual } \\
\text { necesita ayuda? }\end{array}$ \\
\hline
\end{tabular}

${ }^{a}$ Cuestionario tomado de la página web del desarrollador, disponible en: https://www.massgeneral.org/assets/MGH/pdf/psychiatry/psc/ psc-17-english.pdf

${ }^{b}$ Cuestionario tomado de la página web del desarrollador, disponible en: https://www.massgeneral.org/assets/MGH/pdf/psychiatry/psc/ psc-17-spanish.pdf

'Versión del cuestionario correspondiente a la utilizada por la Junta Nacional de Auxilio Escolar y Becas del Ministerio de Educación de Chile en el contexto del Programa Habilidades para la Vida.

Tabla 3. Síntesis de la evaluación de la sección "Instrucciones" (versión para adolescentes) por parte del comité evaluador.

\begin{tabular}{|c|c|c|c|c|c|}
\hline $\begin{array}{c}\text { Original } \\
\text { en inglés }\end{array}$ & $\begin{array}{c}\text { Original en } \\
\text { español }^{\mathrm{b}}\end{array}$ & $\begin{array}{c}\text { Versión Chilena } \\
\text { JUNAEB }^{c}\end{array}$ & $\begin{array}{l}\text { Versión Peruana } \\
\text { Preliminar }\end{array}$ & Observaciones & $\begin{array}{c}\text { Versión } \\
\text { peruana final }\end{array}$ \\
\hline $\begin{array}{l}\text { Please } \\
\text { mark } \\
\text { under the } \\
\text { heading } \\
\text { that best } \\
\text { fits you }\end{array}$ & $\begin{array}{l}\text { Indique cual } \\
\text { síntoma mejor te } \\
\text { describe: }\end{array}$ & $\begin{array}{l}\text { No especifica } \\
\text { indicaciones. }\end{array}$ & $\begin{array}{l}\text { Por favor, marca } \\
\text { lo que mejor te } \\
\text { describa }\end{array}$ & $\begin{array}{l}\text { El comité consideró } \\
\text { por unanimidad que } \\
\text { las instrucciones se } \\
\text { mantengan lo más } \\
\text { similares posible a la } \\
\text { versión original en } \\
\text { inglés por ser más } \\
\text { claras y directas. }\end{array}$ & $\begin{array}{l}\text { Por favor, } \\
\text { marca lo } \\
\text { que mejor te } \\
\text { describa }\end{array}$ \\
\hline
\end{tabular}

${ }^{a}$ Cuestionario tomado de la página web del desarrollador, disponible en: https://www.massgeneral.org/assets/MGH/pdf/psychiatry/psc/ psc-17-english.pdf

${ }^{\mathrm{b}}$ Cuestionario tomado de la página web del desarrollador, disponible en: https://www.massgeneral.org/assets/MGH/pdf/psychiatry/psc/ psc-17-spanish.pdf

'Versión del cuestionario correspondiente a la utilizada por la Junta Nacional de Auxilio Escolar y Becas del Ministerio de Educación de Chile en el contexto del Programa Habilidades para la Vida. 
Tabla 4. Síntesis de la evaluación de los ítems del instrumento PSC (versión para adolescentes) por parte del comité evaluador.

\begin{tabular}{|c|c|c|c|c|c|c|}
\hline $\begin{array}{c}\text { Ítem } \\
\mathrm{N}^{\circ}\end{array}$ & $\begin{array}{l}\text { Original en } \\
\text { inglés }^{\mathrm{a}}\end{array}$ & $\begin{array}{c}\text { Original en } \\
\text { español }^{\mathrm{b}}\end{array}$ & $\begin{array}{c}\text { Versión } \\
\text { Chilena } \\
\text { JUNAEB }\end{array}$ & $\begin{array}{c}\text { Versión } \\
\text { Peruana } \\
\text { Preliminar }\end{array}$ & Observaciones & $\begin{array}{c}\text { Versión } \\
\text { peruana final }\end{array}$ \\
\hline 1 & $\begin{array}{l}\text { Fidgety, unable } \\
\text { to sit still }\end{array}$ & $\begin{array}{l}\text { Eres } \\
\text { inquieto(a) }\end{array}$ & $\begin{array}{l}\text { Eres } \\
\text { inquieto(a) }\end{array}$ & $\begin{array}{l}\text { Eres } \\
\text { inquieto(a) }\end{array}$ & $\begin{array}{l}\text { Se considera que, de } \\
\text { mantenerse únicamente } \\
\text { como "eres inquieto", puede } \\
\text { ser considerado como una } \\
\text { cualidad esperada por lo cual } \\
\text { se añade el adverbio "muy" } \\
\text { para dar una connotación de } \\
\text { mayor intensidad. Se añade } \\
\text { también "tienes demasiada } \\
\text { energía" de manera similar } \\
\text { a la versión para padres y } \\
\text { cuidadores. }\end{array}$ & $\begin{array}{l}\text { Eres muy } \\
\text { inquieto(a), } \\
\text { tienes } \\
\text { demasiada } \\
\text { energía }\end{array}$ \\
\hline 2 & $\begin{array}{l}\text { Feel sad, } \\
\text { unhappy }\end{array}$ & $\begin{array}{l}\text { Te sientes } \\
\text { triste, infeliz }\end{array}$ & $\begin{array}{l}\text { Te sientes } \\
\text { triste, infeliz }\end{array}$ & $\begin{array}{l}\text { Te sientes } \\
\text { triste }\end{array}$ & $\begin{array}{l}\text { Se considera mantener la } \\
\text { versión chilena y omitir la } \\
\text { palabra "infeliz" porque ese } \\
\text { término no es comúnmente } \\
\text { usado para expresar un } \\
\text { estado de ánimo. }\end{array}$ & $\begin{array}{l}\text { Te sientes } \\
\text { triste }\end{array}$ \\
\hline 3 & $\begin{array}{l}\text { Daydream too } \\
\text { much }\end{array}$ & $\begin{array}{l}\text { Eres muy } \\
\text { soñador(a) }\end{array}$ & $\begin{array}{l}\text { Eres muy } \\
\text { soñador(a) }\end{array}$ & $\begin{array}{l}\text { Eres muy } \\
\text { soñador(a) }\end{array}$ & $\begin{array}{l}\text { Se considera modificar a } \\
\text { "sueñas despierto(a)" pues } \\
\text { la frase original ("eres } \\
\text { muy soñador(a)" podría } \\
\text { interpretarse de manera } \\
\text { distinta (p.ej. idealista). Se } \\
\text { añade "estás es tu propio } \\
\text { mundo" para complementar } \\
\text { y favorecer la comprensión. }\end{array}$ & $\begin{array}{l}\text { Sueñas } \\
\text { despierto(a)/ } \\
\text { estás en tu } \\
\text { propio mundo }\end{array}$ \\
\hline 4 & Refuse to share & $\begin{array}{l}\text { Te rehusas a } \\
\text { compartir }\end{array}$ & $\begin{array}{l}\text { Te niegas a } \\
\text { compartir }\end{array}$ & $\begin{array}{l}\text { Te rehusas a } \\
\text { compartir }\end{array}$ & 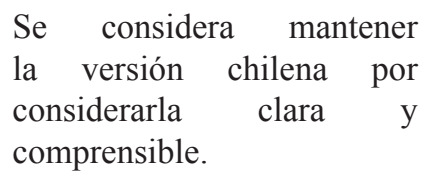 & $\begin{array}{l}\text { Te niegas a } \\
\text { compartir }\end{array}$ \\
\hline 5 & $\begin{array}{l}\text { Do not } \\
\text { understand } \\
\text { other people's } \\
\text { feelings }\end{array}$ & $\begin{array}{l}\text { No } \\
\text { comprendes } \\
\text { los } \\
\text { sentimientos } \\
\text { de otros }\end{array}$ & $\begin{array}{l}\text { No } \\
\text { comprendes } \\
\text { los } \\
\text { sentimientos } \\
\text { de otros }\end{array}$ & $\begin{array}{l}\text { No } \\
\text { comprendes } \\
\text { los } \\
\text { sentimientos } \\
\text { de otros }\end{array}$ & $\begin{array}{l}\text { Se mantiene la versión } \\
\text { original en español y la } \\
\text { chilena con un cambio sutil } \\
\text { en el fraseo, que es una } \\
\text { expresión más comúnmente } \\
\text { usada. }\end{array}$ & $\begin{array}{l}\text { No } \\
\text { comprendes } \\
\text { los } \\
\text { sentimientos } \\
\text { de los demás }\end{array}$ \\
\hline 6 & Feel hopeless & $\begin{array}{l}\text { Te sientes sin } \\
\text { esperanzas }\end{array}$ & $\begin{array}{l}\text { Te sientes sin } \\
\text { esperanzas }\end{array}$ & $\begin{array}{l}\text { Te sientes sin } \\
\text { esperanzas }\end{array}$ & $\begin{array}{l}\text { Se considera usar las } \\
\text { expresiones de la versión } \\
\text { para padres y cuidadores por } \\
\text { considerarlas de más uso y } \\
\text { comprensión por parte de la } \\
\text { población adolescente. }\end{array}$ & $\begin{array}{l}\text { Te sientes } \\
\text { pesimista/ } \\
\text { piensas que } \\
\text { las cosas son } \\
\text { difíciles y te } \\
\text { van a salir } \\
\text { mal }\end{array}$ \\
\hline 7 & $\begin{array}{l}\text { Have trouble } \\
\text { concentrating }\end{array}$ & $\begin{array}{l}\text { Tienes } \\
\text { problemas } \\
\text { para } \\
\text { concentrarte }\end{array}$ & $\begin{array}{l}\text { Tienes } \\
\text { problemas } \\
\text { para } \\
\text { concentrarte }\end{array}$ & $\begin{array}{l}\text { Tienes } \\
\text { problemas } \\
\text { para } \\
\text { concentrarte }\end{array}$ & $\begin{array}{l}\text { Se considera modificar por } \\
\text { "Te cuesta concentrarte" por } \\
\text { ser una expresión más clara y } \\
\text { directa. }\end{array}$ & $\begin{array}{l}\text { Te cuesta } \\
\text { concentrarte }\end{array}$ \\
\hline
\end{tabular}


Tabla 4 (cont.) Síntesis de la evaluación de los ítems del instrumento PSC (versión para adolescentes) por parte del comité evaluador.

\begin{tabular}{|c|c|c|c|c|c|c|}
\hline $\begin{array}{l}\text { Ítem } \\
\mathbf{N}^{\circ}\end{array}$ & $\begin{array}{l}\text { Original en } \\
\text { inglés }^{\mathrm{a}}\end{array}$ & $\begin{array}{c}\text { Original en } \\
\text { español }^{\mathrm{b}}\end{array}$ & $\begin{array}{l}\text { Versión } \\
\text { Chilena } \\
\text { JUNAEB }\end{array}$ & $\begin{array}{c}\text { Versión } \\
\text { Peruana } \\
\text { Preliminar }\end{array}$ & Observaciones & $\begin{array}{c}\text { Versión } \\
\text { peruana final }\end{array}$ \\
\hline 8 & $\begin{array}{l}\text { Fight with } \\
\text { other children }\end{array}$ & $\begin{array}{l}\text { Te peleas } \\
\text { con otros } \\
\text { niños(as) }\end{array}$ & $\begin{array}{l}\text { Te peleas } \\
\text { con otros } \\
\text { adolescentes }\end{array}$ & $\begin{array}{l}\text { Te peleas } \\
\text { con otros } \\
\text { niños(as) }\end{array}$ & $\begin{array}{l}\text { Se sustituye "niños(as)" por } \\
\text { "chicos(as)" y se mantiene } \\
\text { "adolescentes". }\end{array}$ & $\begin{array}{l}\text { Peleas } \\
\text { con otros } \\
\text { chicos(as) o } \\
\text { adolescentes }\end{array}$ \\
\hline 9 & $\begin{array}{l}\text { Down on } \\
\text { yourself }\end{array}$ & $\begin{array}{l}\text { Te criticas a } \\
\text { ti mismo(a) }\end{array}$ & $\begin{array}{l}\text { Te criticas a } \\
\text { ti mismo(a) }\end{array}$ & $\begin{array}{l}\text { Te criticas a ti } \\
\text { mismo(a) }\end{array}$ & $\begin{array}{l}\text { La expresión "te criticas a ti } \\
\text { mismo(a)" podría ser menos } \\
\text { usada por la población } \\
\text { infantojuvenil. El comité } \\
\text { considera mantener la } \\
\text { expresión "Te sientes mal } \\
\text { contigo mismo(a)" para } \\
\text { mantener la idea del ítem } \\
\text { original que se refiere a la } \\
\text { evaluación en relación con } \\
\text { uno mismo. }\end{array}$ & $\begin{array}{l}\text { Te sientes } \\
\text { mal contigo } \\
\text { mismo(a) }\end{array}$ \\
\hline 10 & $\begin{array}{l}\text { Blame others } \\
\text { for your } \\
\text { troubles }\end{array}$ & $\begin{array}{l}\text { Culpas a } \\
\text { otros por tus } \\
\text { problemas }\end{array}$ & $\begin{array}{l}\text { Culpas a } \\
\text { otros por tus } \\
\text { problemas }\end{array}$ & $\begin{array}{l}\text { Culpas a } \\
\text { otros por tus } \\
\text { problemas }\end{array}$ & $\begin{array}{l}\text { Se mantiene la versión } \\
\text { original en español y la } \\
\text { chilena con un cambio sutil } \\
\text { en el fraseo, que es una } \\
\text { expresión más comúnmente } \\
\text { usada. }\end{array}$ & $\begin{array}{l}\text { Culpas a los } \\
\text { demás de tus } \\
\text { problemas }\end{array}$ \\
\hline 11 & $\begin{array}{l}\text { Seem to be } \\
\text { having less fun }\end{array}$ & $\begin{array}{l}\text { Parece que } \\
\text { te diviertes } \\
\text { menos }\end{array}$ & $\begin{array}{l}\text { Parece que } \\
\text { te diviertes } \\
\text { menos que } \\
\text { antes }\end{array}$ & $\begin{array}{l}\text { Parece que } \\
\text { te diviertes } \\
\text { menos }\end{array}$ & $\begin{array}{l}\text { Se considera mantener } \\
\text { la versión chilena por } \\
\text { considerarla clara y } \\
\text { comprensible y se añade la } \\
\text { forma utilizada en la versión } \\
\text { para padres y cuidadores } \\
\text { porque también puede } \\
\text { comprenderse con facilidad } \\
\text { por la población adolescente. }\end{array}$ & $\begin{array}{l}\text { Parece que } \\
\text { te diviertes } \\
\text { menos que } \\
\text { antes/ Te } \\
\text { aburres } \\
\text { fácilmente }\end{array}$ \\
\hline 12 & $\begin{array}{l}\text { Do not listen } \\
\text { to rules }\end{array}$ & $\begin{array}{l}\text { No obedeces } \\
\text { reglas }\end{array}$ & $\begin{array}{l}\text { Eres } \\
\text { desobediente } \\
\text { con las } \\
\text { reglas. }\end{array}$ & $\begin{array}{l}\text { No obedeces } \\
\text { reglas }\end{array}$ & $\begin{array}{l}\text { Se reformula la versión } \\
\text { original en español para } \\
\text { expresar que el ítem no trata } \\
\text { únicamente de obedecer } \\
\text { reglas, sino de no seguir } \\
\text { órdenes entendidas como } \\
\text { mandatos o indicaciones } \\
\text { además de cumplir las reglas. }\end{array}$ & $\begin{array}{l}\text { No sigues } \\
\text { las órdenes/ } \\
\text { reglas }\end{array}$ \\
\hline 13 & $\begin{array}{l}\text { Act as if } \\
\text { driven by a } \\
\text { motor }\end{array}$ & $\begin{array}{l}\text { Eres } \\
\text { incansable }\end{array}$ & $\begin{array}{l}\text { Te cuesta } \\
\text { mucho } \\
\text { cansarte, } \\
\text { tienes mucha } \\
\text { energía }\end{array}$ & $\begin{array}{l}\text { Eres } \\
\text { incansable }\end{array}$ & $\begin{array}{l}\text { El comité considera usar } \\
\text { la frase "Te cuesta estar } \\
\text { tranquilo(a)" por ser más } \\
\text { entendible y usada por la } \\
\text { población adolescente en } \\
\text { comparación a la propuesta } \\
\text { original. }\end{array}$ & $\begin{array}{l}\text { Te cuesta } \\
\text { estar } \\
\text { tranquilo(a) }\end{array}$ \\
\hline
\end{tabular}


Tabla 4 (cont.) Síntesis de la evaluación de los ítems del instrumento PSC (versión para adolescentes) por parte del comité evaluador.

\begin{tabular}{|c|c|c|c|c|c|c|}
\hline $\begin{array}{l}\text { Ítem } \\
\mathrm{N}^{\circ}\end{array}$ & $\begin{array}{l}\text { Original en } \\
\text { inglés }^{\mathrm{a}}\end{array}$ & $\begin{array}{c}\text { Original en } \\
\text { español }^{\mathrm{b}}\end{array}$ & $\begin{array}{c}\text { Versión } \\
\text { Chilena } \\
\text { JUNAEB }\end{array}$ & $\begin{array}{c}\text { Versión } \\
\text { Peruana } \\
\text { Preliminar }\end{array}$ & Observaciones & $\begin{array}{c}\text { Versión } \\
\text { peruana final }\end{array}$ \\
\hline 14 & Tease others & $\begin{array}{l}\text { Molestas a } \\
\text { otros }\end{array}$ & $\begin{array}{l}\text { Molestas } \\
\text { a otras } \\
\text { personas }\end{array}$ & $\begin{array}{l}\text { Molestas a } \\
\text { otros }\end{array}$ & $\begin{array}{l}\text { Se añade "te burlas" para } \\
\text { aportar una mayor claridad al } \\
\text { ítem y se modifica sutilmente } \\
\text { el fraseo (de "a otros" a } \\
\text { "los demás") ya que es una } \\
\text { expresión más comúnmente } \\
\text { usada y para mantener } \\
\text { congruencia con los ítems } \\
\text { previos. }\end{array}$ & $\begin{array}{l}\text { Molestas o te } \\
\text { burlas de los } \\
\text { demás }\end{array}$ \\
\hline 15 & Worry a lot & $\begin{array}{l}\text { Te preocupas } \\
\text { mucho }\end{array}$ & $\begin{array}{l}\text { Te preocupas } \\
\text { mucho }\end{array}$ & $\begin{array}{l}\text { Te preocupas } \\
\text { mucho }\end{array}$ & $\begin{array}{l}\text { Se considera mantener la } \\
\text { versión original y chilena } \\
\text { por considerarla clara y } \\
\text { comprensible. }\end{array}$ & $\begin{array}{l}\text { Te preocupas } \\
\text { mucho }\end{array}$ \\
\hline 16 & $\begin{array}{l}\text { Take things } \\
\text { that do not } \\
\text { belong to you }\end{array}$ & $\begin{array}{l}\text { Tomas cosas } \\
\text { que no te } \\
\text { pertenecen }\end{array}$ & $\begin{array}{l}\text { Tomas cosas } \\
\text { que no te } \\
\text { pertenecen }\end{array}$ & $\begin{array}{l}\text { Tomas cosas } \\
\text { que no te } \\
\text { pertenecen }\end{array}$ & $\begin{array}{l}\text { De manera similar a la versión } \\
\text { para padres y cuidadores, se } \\
\text { optó por el término "coge" } \\
\text { porque tiene una connotación } \\
\text { más definitiva o permanente } \\
\text { (por ejemplo, "coge plata"), } \\
\text { además de que es una palabra } \\
\text { frecuentemente utilizada } \\
\text { en el contexto peruano en } \\
\text { comparación a "tomar" } \\
\text { (objetos). Se reformuló el } \\
\text { ítem. }\end{array}$ & $\begin{array}{l}\text { Coges cosas } \\
\text { ajenas }\end{array}$ \\
\hline 17 & Distract easily & $\begin{array}{l}\text { Te distraes } \\
\text { fácilmente }\end{array}$ & $\begin{array}{l}\text { Te distraes } \\
\text { fácilmente }\end{array}$ & $\begin{array}{l}\text { Te distraes } \\
\text { fácilmente }\end{array}$ & $\begin{array}{l}\text { Se considera mantener la } \\
\text { versión original y chilena } \\
\text { por considerarla clara y } \\
\text { comprensible. }\end{array}$ & $\begin{array}{l}\text { Te distraes } \\
\text { fácilmente }\end{array}$ \\
\hline
\end{tabular}

${ }^{a}$ Cuestionario tomado de la página web del desarrollador, disponible en: https://www.massgeneral.org/assets/MGH/pdf/psychiatry/psc/ psc-17-english.pdf

${ }^{\mathrm{b}}$ Cuestionario tomado de la página web del desarrollador, disponible en: https://www.massgeneral.org/assets/MGH/pdf/psychiatry/psc/ psc-17-spanish.pdf

'Versión del cuestionario correspondiente a la utilizada por la Junta Nacional de Auxilio Escolar y Becas del Ministerio de Educación de Chile en el contexto del Programa Habilidades para la Vida.

\section{Versión para adolescentes}

En relación con las instrucciones, también se consideró que sean breves y directas tal como la versión en inglés. Los ítems 15 (preocupación) y 17 (distraibilidad) se han mantenido igual en la versión peruana final en relación con las otras versiones en español.

Los ítems 5 (empatía), 7 (concentración), 8 (peleas), 10 (culpar a otros) y 14 (molestar a otros) presentan modificaciones sutiles respecto a la versión original en español y/o la versión chilena. En el ítem 2 (tristeza) se retiró una palabra en la versión peruana final con respecto a las versiones original en español y chilena.

Los ítems 1 (hiperactividad), 3 (soñar despierto), 6 (desesperanza), 11 (anhedonia) y 12 (desobediencia) se modificaron añadiendo expresiones o palabras similares a las usadas en la versión para padres y cuidadores, con modificaciones importantes con relación a las versiones original y chilena, mientras 
Tabla 5. Síntesis de la evaluación de las opciones de respuesta del instrumento PSC (versión para padres y cuidadores y versión para adolescentes) por parte del comité evaluador.

\begin{tabular}{llllll}
\hline $\begin{array}{c}\text { Original } \\
\text { en inglés }^{\mathrm{a}}\end{array}$ & $\begin{array}{c}\text { Original en } \\
\text { español }^{\mathrm{b}}\end{array}$ & $\begin{array}{c}\text { Versión } \\
\text { Chilena } \\
\text { JUNAEB }\end{array}$ & $\begin{array}{c}\text { Versión Peruana } \\
\text { Preliminar }\end{array}$ & \multicolumn{1}{c}{ Observaciones } & $\begin{array}{c}\text { Versión peruana } \\
\text { final }\end{array}$ \\
\hline Never & Nunca & Nunca & Nunca & $\begin{array}{l}\text { Por unanimidad se } \\
\text { consideró mantenerla igual } \\
\text { que en la versión original } \\
\text { en español y la chilena. }\end{array}$ & Nunca \\
Sometimes & Algunas veces & A veces & A veces & $\begin{array}{l}\text { Por unanimidad se } \\
\text { consideró mantenerla igual } \\
\text { que en la versión chilena. }\end{array}$ & A veces \\
& Frecuentemente & Seguido & Frecuentemente & $\begin{array}{l}\text { Por unanimidad se } \\
\text { consideró mantenerla igual } \\
\text { que en la versión original } \\
\text { en español. }\end{array}$ & Frecuentemente \\
\hline
\end{tabular}

$\overline{{ }^{a} \text { Cuestionario tomado de la página web del desarrollador, disponible en: https://www.massgeneral.org/assets/MGH/pdf/ }}$ psychiatry/psc/psc-17-english.pdf

${ }^{\mathrm{b}}$ Cuestionario tomado de la página web del desarrollador, disponible en: https://www.massgeneral.org/assets/MGH/pdf/ psychiatry/psc/psc-17-spanish.pdf

${ }^{c}$ Versión del cuestionario correspondiente a la utilizada por la Junta Nacional de Auxilio Escolar y Becas del Ministerio de Educación de Chile en el contexto del Programa Habilidades para la Vida.

que los ítems 9 (autoestima), 13 (intranquilidad) y 16 (coge cosas ajenas) tuvieron cambios significativos con respecto a las versiones original en español y chilena.

En el ítem 4 (negarse a compartir) se mantuvo la versión chilena.

En relación con las 3 alternativas de respuesta para cada ítem se prefirió la opción "frecuentemente" de la versión original, la alternativa "a veces" de la versión chilena y se conservó la opción "nunca" de ambas versiones (original en español y chilena), tanto en la versión de padres y cuidadores como en la de adolescentes.

\section{DISCUSIÓN}

El trabajo realizado mediante juicio de expertos permitió obtener una primera versión peruana del instrumento PSC-17. De manera similar a la adaptación realizada en Chile sobre el instrumento original (PSC35), el presente trabajo realizó modificaciones en los ítems para ser comprensibles y aplicables en nuestro contexto. Sin embargo, la metodología propuesta en aquel trabajo, a diferencia del presente, incluyó la evaluación por padres como jueces (15). La traducción inversa al idioma original (inglés) es un paso recomendado en el proceso de adaptación cultural de instrumentos psicométricos (16), que no realizamos considerando que ya existen versiones en español (7). La presente adaptación significa un esfuerzo por contar con un instrumento que ayude en la detección de problemas de salud mental en niños y adolescentes en un contexto de mayor vulnerabilidad como es el de la pandemia por COVID-19.

Entre las limitaciones de este trabajo tenemos que solamente se ha centrado en el aspecto cualitativo y no se han determinado indicadores cuantitativos sobre la opinión de los jueces sobre claridad, pertinencia y relevancia tales como la $\mathrm{V}$ de Aiken. Aunque la versión peruana obtenida requiere ser evaluada en sus características psicométricas para determinar que sean adecuadas y exhiba la confiabilidad y validez del instrumento original, el presente trabajo busca dar un aporte inicial al campo de la salud mental infantojuvenil poniendo a disposición de la comunidad científica peruana y del personal de salud un instrumento que ayude en la identificación oportuna de problemas de salud mental en esta población. De esta manera, el instrumento puede ser de utilidad clínica, pero también a nivel de la investigación. Asimismo, el presente reporte pretende incentivar el desarrollo de más investigaciones sobre la utilidad de este tipo de instrumentos en nuestro medio y contribuir a mejorar los procesos de detección e intervención en niños, niñas y adolescentes con problemas de salud mental. 


\section{Contribuciones de los autores:}

CCV, JCP y JVD aportaron la idea y diseño del estudio. $\mathrm{CCV}$ recogió los datos. $\mathrm{CCV}$, JCP y JVD analizaron e interpretaron datos. $\mathrm{CCV}$ realizó la redacción del borrador del artículo. CCV, JCP y JVD realizaron aportes al borrador e hicieron una revisión crítica del contenido intelectual. CCV y JVD realizaron la revisión iterativa del manuscrito. Todos los autores aprobaron la versión final del manuscrito.

\section{Correspondencia:}

Carla P. Cortez Vergara

Correo electrónico: carla.cortez.v@upch.pe

Financiamiento: Autofinanciado.

Conflictos de interés: Los autores declaran no tener conflictos de interés.

\section{REFERENCIAS BIBLIOGRÁFICAS}

1. Meherali S, Punjani N, Louie-Poon S, Abdul Rahim K, Das JK, Salam R, et al. Mental health of children and adolescents amidst COVID-19 and Past Pandemics: A rapid systematic review. Int J Environ Res Public Health. 2004;1(1):1-2.

2. Loades ME, Chatburn E, Higson-Sweeney N, Reynolds S, Shafran R, Brigden A, et al. Rapid Systematic Review: The impact of social isolation and loneliness on the mental health of children and adolescents in the context of COVID-19. J Am Acad Child Adolesc Psychiatry. 2020; 59(11): 1218-1239.

3. Shonkoff JP, Garner AS, Siegel BS, Dobbins MI, Earls MF, et al. The Lifelong Effects of Early Childhood Adversity and Toxic Stress. Pediatrics. 2012;129(1):e232-46.

4. Instituto Nacional de Salud Mental. Estudio epidemiológico de salud mental de niños y adolescentes en Lima Metropolitana y Callao del 2007. Anales de Salud Mental. 2012; 28(1).

5. Nakamura BJ, Ebesutani C, Bernstein A, Chorpita BF. A Psychometric Analysis of the Child Behavior Checklist DSM-Oriented Scales. J Psychopathol Behav Assess. 2009;31(3):178-89.

6. Warnick EM, Bracken MB, Kasl S; Centre for Reviews and Dissemination (UK). Screening efficiency of the child behavior checklist and strengths and difficulties questionnaire: a systematic review. Child Adolesc Ment Health. 2008; 13(3): 140-147.

7. Massachusetts General Hospital. Pediatric Symptom Checklist. Massachusetts: Massachusetts General Hospital. (Citado el 3 de junio de 2021). Disponible en: https://www.massgeneral.org/psychiatry/ treatments-and-services/pediatric-symptom-checklist

8. Liu J, Guo S, Gao R, DiStefano C. Investigating school children's behavioral and emotional problems using pediatric symptoms checklist-17 in a structural equation modeling framework. Sch Psychol Int. 2020;41(3):257-75.

9. Bernal P, Bendell Estroff D, Aboudarham JF, Murphy M, Keller A, Jellinek MS. Psychosocial Morbidity: The Economic Burden in a Pediatric Health Maintenance Organization Sample. Arch Pediatr Adolesc Med. 2000;154(3):261.

10. Guzmán J, Kessler RC, Squicciarini AM, George M, Baer L, Canenguez KM, et al. Evidence for the Effectiveness of a National School-Based Mental Health Program in Chile. J Am Acad Child Adolesc Psychiatry. 2015;54(10):799-807.

11. Murphy JM, Bergmann P, Chiang C, Sturner R, Howard B, Abel MR, et al. The PSC-17: Subscale Scores, Reliability, and Factor Structure in a New National Sample. Pediatrics. 2016;138(3):e20160038-e20160038.

12. Leiva L, Rojas R, Peña F, Vargas B, Scquicciarini A. Detectando las Dificultades Emocionales y Conductuales en la Escuela: Validación de PSC-17. Rev Iberoam Diagn Ev. 2019; 1(50), 95-105.

13. Gaite L, Ramírez N, Herrera S, Vázquez JL. Traducción y adaptación transcultural de instrumentos de evaluación en psiquiatría: aspectos metodológicos. Arch. Neurobiol. 60(2): 91-111.

14. Ministerio de Educación. Detección de Riesgo Psicosocial. PSC17 y PSC Y17. Proceso adecuación e implementación 2020 del Programa Habilidades para la Vida. Lima: Ministerio de Educación; 2020.

15. George M, Siraqyan X, Mores R, De La Barra F, Rodríguez J, López C, et al. Adaptación y validación de dos instrumentos de pesquisa de problemas de salud mental de escolares de $1^{\circ}$ básico. Revista de Psicología. 1994; 5(26).

16. Ramada-Rodilla JM, Serra-Pujadas C, DelclósClanchet GL. Adaptación cultural y validación de cuestionarios de salud: revisión y recomendaciones metodológicas. Salud Publ Mex. 2013;55(1):57-66.
Recibido: 05/10/2021

Aceptado: 30/12/2021 